\title{
The Pattern of Cardiac Disease at Coronary Care Unit of Dhaka Medical College Hospital
}

\author{
Abdul Wadud Chowdhury ${ }^{1}$, Nur Alam², HI Lutfur Rahman Khan ${ }^{1}$, Khandker Md. Nurus Sabah ${ }^{1}$, \\ Mohammad Gaffar Amin ${ }^{1}$ \\ ${ }^{1}$ Department of Cardiology, Dhaka Medical College,Dhaka, ${ }^{2}$ Department of Cardiology, National \\ Institute of Cardiovascular Diseases, Dhakal
}

Key Words :
Cardiovascular
diseases,
Coronary care
unit.

\begin{abstract}
:
\end{abstract}
Background: Epidemiological transition which is taking place in every part of the world, among all races, ethnic groups and cultures has resulted in the global rise in cardiovascular disease (CVD). Cardiovascular disease is the leading cause of morbidity and mortality throughout the world. We tried to assess the pattern of cardiac disease at coronary care unit (CCU) of tertiary care hospital.

Methods: A cross sectional study conducted among the patients admitted at CCU of Dhaka Medical College Hospital (DMCH) during the period of 1st January 2010 to 31st December 2010. A total of 2415 patients were included in the study.

Results: A total 2415 patients in the year 2010, were admitted at CCU of DMCH, among them 56\% $(n=1346)$ were male and $44 \%(n=1069)$ were female. Out of total admitted patients $220(9 \%)$ were below 30 years of age and $588(24 \%) \& 1607$ (67\%) were the age group of 31 to 44 years and 45 years and above age group respectively. Among the disease group ischemic heart disease( IHD) (45\%) was the most common cause of hospitalization followed by heart failure (HF) (16\%) valvular heart disease (9\%), hypertension (7\%) and arrhythmia (2\%). Acute myocardial infarction (30\%) was the leading cause of IHD followed by unstable angina (15\%).

Conclusion: This study has found that almost three-quarters of cardiac disease were due to IHD, $H F$ and valvular heart disease in decreasing order of frequency. Countries like Bangladesh can be able to spend very little resources to address those cardiovascular diseases. Along with public as well as private sector efforts, public awareness about the risk factors for cardiovascular diseases should be improved to reduce this sort mortality and morbidity.

(Cardiovasc. j. 2015; 7(2): 119-122)

\section{Introduction:}

Epidemiological transition which is taking place in every part of the world, among all races, ethnic groups and cultures has resulted in the global rise in cardiovascular disease (CVD). Cardiovascular disease is the leading cause of morbidity and mortality throughout the world. It has no geographic, gender or economic boundaries. Today CVD became the most common cause of death accounting for $30 \%$ of death worldwide, with $80 \%$ of the burden now occurring in developing countries. ${ }^{1,2}$

At present Bangladesh is facing a dual burden of communicable and non- communicable disease. Among the non-communicable diseases CVD evolve from the complex interaction of multiple determinants and risk factors. Interventions need to address this determinants and risk factors simultaneously, integrating policy and public health interventions that target entire population as well as high risk individuals and those with early or established disease. ${ }^{3}$

Determining the current pattern of cardiac disease in our setting is required which can also be considered as the beginning of efforts to put forward in characterization of the most prevailing cardiac diseases in our setting and ultimately improve its detection, treatment and prevention. Therefore this study was carried out to describe the pattern of cardiac diseases among patients who were admitted at coronary care unit (CCU) of Dhaka Medical College Hospital (DMCH).

\section{Methods:}

This was a cross sectional study conducted among the patients admitted at CCU of Dhaka Medical College Hospital (DMCH) during the period of 1st

Address of Correspondence: Dr. Nur Alam, Department of Cardiology, National Institute of Cardiovascular Diseases, Dhaka, Bangladesh. email : alamnur28@yahoo.com 
January 2010 to 31st December 2010. A total of 2415 patients were included in the study.

The inclusion criteria were all the patients admitted at CCU of Dhaka Medical College Hospital $(\mathrm{DMCH})$ during the study period. Patients are diagnosed according to latest definition of cardiac diseases. Myocardial infarction includes both ST segment elevated MI and Non ST segment elevated myocardial infarction. The patients of heart failure only due to cardiac causes were included. The patient category 'others' included congenital heart disease (other than associated arrhythmia and heart failure), cardiac tumors, non specific chest pain etc. Data were collected using structured pretested format. The collected data were analyzed by Statistical Package for Social Science (SPSS) for windows version 12.0. Descriptive analysis was made to describe the pattern of disease. Results were then presented by tables and diagrams. Prior to commencement of this study the respective authority approved the research protocol. Ethical clearance was obtained from the department and institution concerned for this study.

\section{Results:}

In the year 2010 , a total 2415 patients were admitted at CCU of DMCH. Among them 56\% $(n=1346)$ were male and $44 \%(n=1069)$ were female. Out of total admitted patients $220(9 \%)$ were below 30 years of age and 588 ( $24 \%) \& 1607$ ( $67 \%$ ) were the age group of 31 - 44 years and $\geq 45$ years age group respectively. Among the disease group IHD (45\%) was the most common cause of hospitalization followed by heart failure (HF) (16\%), vulvular heart disease (9\%), hypertension (7\%) and arrhythmia (2\%). Acute myocardial infarction (30\%) was the leading cause of IHD followed by unstable angina (15\%) (Table I).

\section{Table-I}

Pattern of disease of the patients admitted in $C C U$ of $D M C H(n=2415)$

\begin{tabular}{lcc}
\hline Name & $\begin{array}{c}\text { Patient } \\
\text { Number }\end{array}$ & Percentage \\
\hline Myocardial Infarction & 722 & 30 \\
Unstable Angina & 369 & 15 \\
Heart Failure & 380 & 16 \\
Hypertension (Uncontrolled) & 165 & 7 \\
Valvular Heart Disease & 221 & 9 \\
Arrhythmia & 60 & 2 \\
Chronic Stable Angina (CSA) & 44 & 1.8 \\
Pericardial Disease & 26 & 1.07 \\
Cardiomyopathy & 59 & 2 \\
Others & 369 & 15 \\
\hline
\end{tabular}

\section{Table-II}

Age distribution of the study patients

\begin{tabular}{lcc}
\hline Age Group & Patient Number & Percentage \\
\hline$\leq 30$ Years & 220 & $9 \%$ \\
$31-44$ Years & 588 & $24 \%$ \\
$\geq 44$ Years & 1607 & $67 \%$ \\
\hline
\end{tabular}

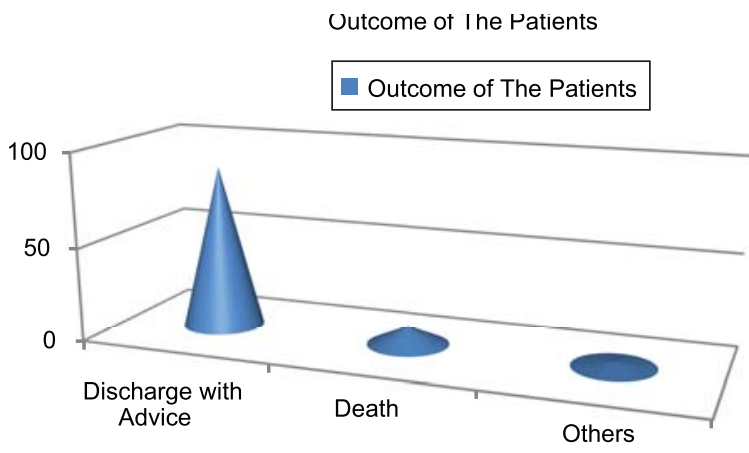

Fig.-1: Diagram shows outcome of the study patients.

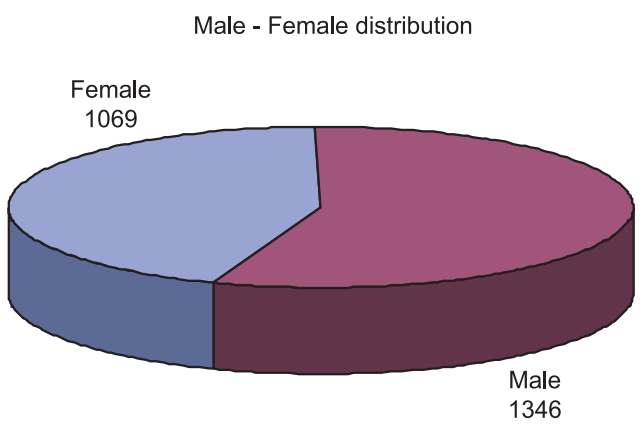

Fig.-2 : Pie diagram showing the sex distribution of the study patients.

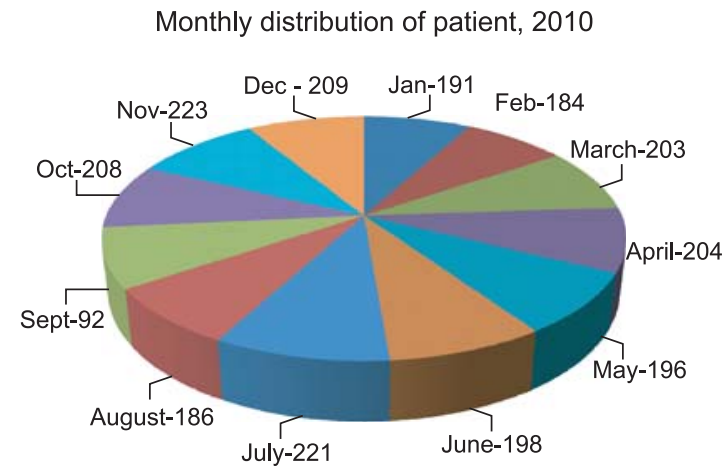

Fig.-3: Monthly distribution of study patients 


\section{Discussion:}

The study was carried out to assess the pattern of cardiac disease in the context of ongoing global epidemiological transition to which Bangladesh is no exception. The pattern of cardiac disease at $\mathrm{DMCH}$ where this study is conducted was assessed 1 year back on a sample of 2169 patients. ${ }^{4}$ The study reported that $78 \%$ of cardiac patients were having IHD, which should have raised an alarm of bell for further studies which need to include screening of high risk individuals so that actual disease prevalence could have been better estimated and subsequent measure could have been taken.

The leading four cardiac disease etiologies identified in this study were IHD, Heart Failure (HF), chronic rheumatic valvular heart disease and hypertensive emergencies. The finding is similar as compared with the studies done in Ethiopia and Malawi and India. ${ }^{5-8}$ In the study conducted on the same setting 1 year back, the pattern of cardiac disease in order of decreasing frequency was IHD, $\mathrm{HF}$ and arrhythmia. The difference could be partly due to- in previous study valvular heart disease and hypertension were included in heart failure heading.

Another study done in Jimma University Specialized Hospital (JUSH), Ethiopia, valvular heart disease was found to be leading cause of cardiac disease. But in the present study IHD is the leading cause. The difference could be due to difference in case definition and sample size. ${ }^{9,10}$

In the present study $9 \%$ of the patients were below 30 years of age which is consistent with the studies in Gondar, (mean age of development of cardiac disease - 23 years), in Tikur Anbesa Hospital (25.5 years) and in Nigeria (24 years). $6,11,12$

In this study, number of female (44\%) patients was comparatively less than that of male (56\%). One important reason for this difference is that less female patients come to hospital for treatment in our country for various socio-economic reasons. This observation is also consistent with the study done in JUSH (female - 47.6\%, male - 52.4\%).

Hypertensive emergencies accounted for $7 \%$ of cardiac patients and it was the 5 th commonest type of heart disease. In other studies done in developing countries hypertension has become the 1st or 2 nd leading cause of heart disease. ${ }^{6,7,13,14}$ However many patients presented with IHD and HF had hypertension as risk factor in the present study.

In the present study a total of 59 patients $(2 \%)$ presented with cardiomyopathy. Similar findings were noted from studies done in developing countries. ${ }^{6,8,10}$.

Majority of the patients (69\%) in the present study had at least one of the conventional risk factors (hypertension, diabetes mellitus, smoking, dyslipidaemia) of cardiovascular disease. Therefore identification of cardiovascular disease risk factors and determination of its overall prevalence in our community is required so that both individualized and community based interventions could be implemented. $.^{4}, 10$

\section{Conclusion:}

This study has found that almost three-quarters of cardiac disease were due to IHD, HF and valvular heart disease in decreasing order of frequency. The proportion of patients with hypertension as cause or contributing factor for cardiac disease is alarming too. Countries like Bangladesh can be able to spend very little resources to address those cardiovascular diseases. Government as well as private sector efforts are needed for public awareness about the risk factors for cardiovascular diseases and mortality and morbidity.

\section{Conflict of Interest - None.}

\section{References:}

1. Gaziano T. Global burden of cardiovascular disease In: Douglas L. Mann, Douglas P. Zipes, Peter Libby, Robert Bonow. Eds. Braunwald's Heart Disease: A Textbook of Cardiovascular Medicine, 8th ed. Philadelphia: Elsevier Saunders,2007:1-21.

2. Gaziano T. Cardiovascular disease in the developing world and its cost effective management, Circulation 2005; 112(23):3547-3553.

3. Ministry of Health and Family Welfare, Government of People's Republic of Bangladesh. Strategic plan for Surveillance and Prevention of Noncomunicable Disease in Bangladesh 2011-2014. 2010, Dhaka.

4. Chowdhury AW, Islam AEM, Siddiq AB et al. Who are the cardiac patients? One year experience in cardiology department of DMCH. 21st Annual conference \& scientific seminar, Association of Physicians of Bangladesh 2010. 
5. Nandi K, Kumar S, Bidhu N et al. Patterns of Ischaemic Heart Disease in a private hospital in India. Cardiocare 1998;64(6):336-337.

6. Hodes M. Patterns of heart disease in Ethiopia as seen in cardiology referral clinic. Cardiology 1988;75(6):456464.

7. Maru M. The changing pattern of cardiovascular disease in Ethiopia. E Afr Med J 1993; 70(12):772-776.

8. Soliman Z, Juma H. Cardiac disease pattern in Northern Malawi: epidemiologic transition perspective. Epidemiology 2008; 18(5):204-208.

9. Habte B, Alemseged F, Tesfaye D. The Pattern of cardiac disease at the cardiac clinic of Jimma university specialized hospital, South West Ethiopia. Ethiop $J$ Health Sci 2010; 20(2): 99.
10. Petros G: Patterns of heart disease in Jimma Hospital. Bulletin of JIHS 1996; 6(2):85-92.

11. Melka A. Rheumatic heart disease in Gondar college of medical sciences teaching hospital: Socio-demographic and clinical profile. Ethiopian Med $J$ 1996;34:207-217.

12. Sani U, Karaye M, Borodo M. Prevalence and pattern of rheumatic heart disease in the Nigerian savannah: an echocardiographic study. Cardiovasc J Afr 2007; 18(5):295-299.

13. Mamo Y, Oli K. Trends of acute myocardial infarction admissions over a decade, Tikur Anbesa Hospital. Ethiopian Med J 2001;39: 193-203.

14. Amoah G, Kallen C, Eitiology of heart failure as seen from a cardiac referral centre in Africa. Cardiology 2000; 93(1-2):11-18. 